\title{
Virtual Reality Models and Digital Engineering Solutions for Technology Transfer
}

\author{
Marco Schumann ${ }^{1}$, Stefan Leye ${ }^{2}$, Artyom Popov ${ }^{3}$, \\ ${ }^{1,2}$ Fraunhofer Institute of Factory Operation and Automation IFF Magdeburg, Germany, \\ ${ }^{3}$ JSC Astana Innovations, Kazakhstan
}

\begin{abstract}
Effective applied research is based on close collaboration between research and industry, which taking the findings of basic research on customer demands as its starting point, creates a new means for developing and marketing innovative products. What is more, growing demands for innovative and sustainable results of research and development are prompting the examination of global trends such as demographic change, growing megacities, rising energy consumption and increasing traffic and the resultant social challenges. These trends and increasing traffic in particular are giving rise to new fields of work, especially for digital technologies, as a social responsibility, e.g., in driver assistance and traffic control systems that increase safety.

This paper examines a means by which digital engineering, virtual and augmented reality technologies can support the creation of sustainable smart manufacturing and smart logistics processes as well as on-the-job training and qualification and knowledge transfer.
\end{abstract}

Keywords - Augmented reality, digital engineering, global trends, logistics, production, social challenges, virtual reality.

\section{INTRODUCTION}

Applied research on behalf of business and society is frequently geared toward individual clients' current, foreseeable and future needs. However, to assure the sustainability of its results, viable applied research will have to be oriented toward the global trends and resultant social challenges in the coming years and decades.

Experiences in Germany have revealed that intensive networking of industrial, basic and applied research and higher education establishes a research environment that supports the entire life cycle of innovative products from their development, manufacture, marketing and use through recycling (Fig. 1). Universities assume the task of suitably assimilating research findings in research and teaching and, thus, contribute to assuring their sustainability and to educating young researchers by systematically developing academic and qualification programs.

Though, this does not happen automatically. New forms and processes of collaboration among everyone involved are required, thus making technology development doubly important: Innovative technologies are needed as an integral part of new products as well as supporting the life cycle of innovative products throughout their development, manufacture and use.

The automotive industry is a prime example. On the one hand, its products - motor vehicles - have become inconceivable without integrated digital technologies and, on the other hand, the development, manufacture and maintenance of vehicles have become inconceivable without digital engineering.

\section{Digital engineering for knowledge transfer in innovation networks}

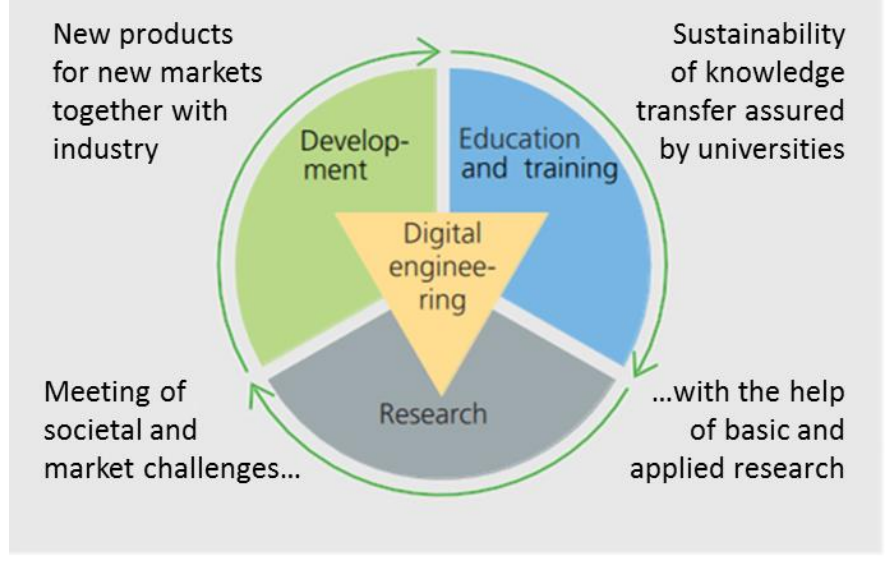

Fig. 1. Digital engineering for knowledge transfer, CFraunhofer IFF.

This trend is ongoing. An integral part of digital engineering, virtual and augmented reality technologies are assuming a key role in different forms of communication. Although virtual and augmented reality and simulation systems were developed, used and marketed largely independently from one another in the past, the future trend is toward integrated engineering platforms that combine real and virtual work systems as well as information and communications technologies with application domains [1] $-[3]$.

The distinctive feature of such platforms is their support of technical, semantic and organizational interoperability with different digital technologies and their facilitation of processdriven forms of interaction between humans and machines, machines and machines, and humans and humans. The focus on current and future fields of application is a significant factor for successful development of sustainable technologies.

Nevertheless, technology development strategies are frequently still developed by research organizations and universities in their role as a "provider" and oriented - if at all only toward their own circle of clients. How can long-range megatrends, e.g., population growth, the depletion of raw materials, urbanization, etc., be incorporated in new market 

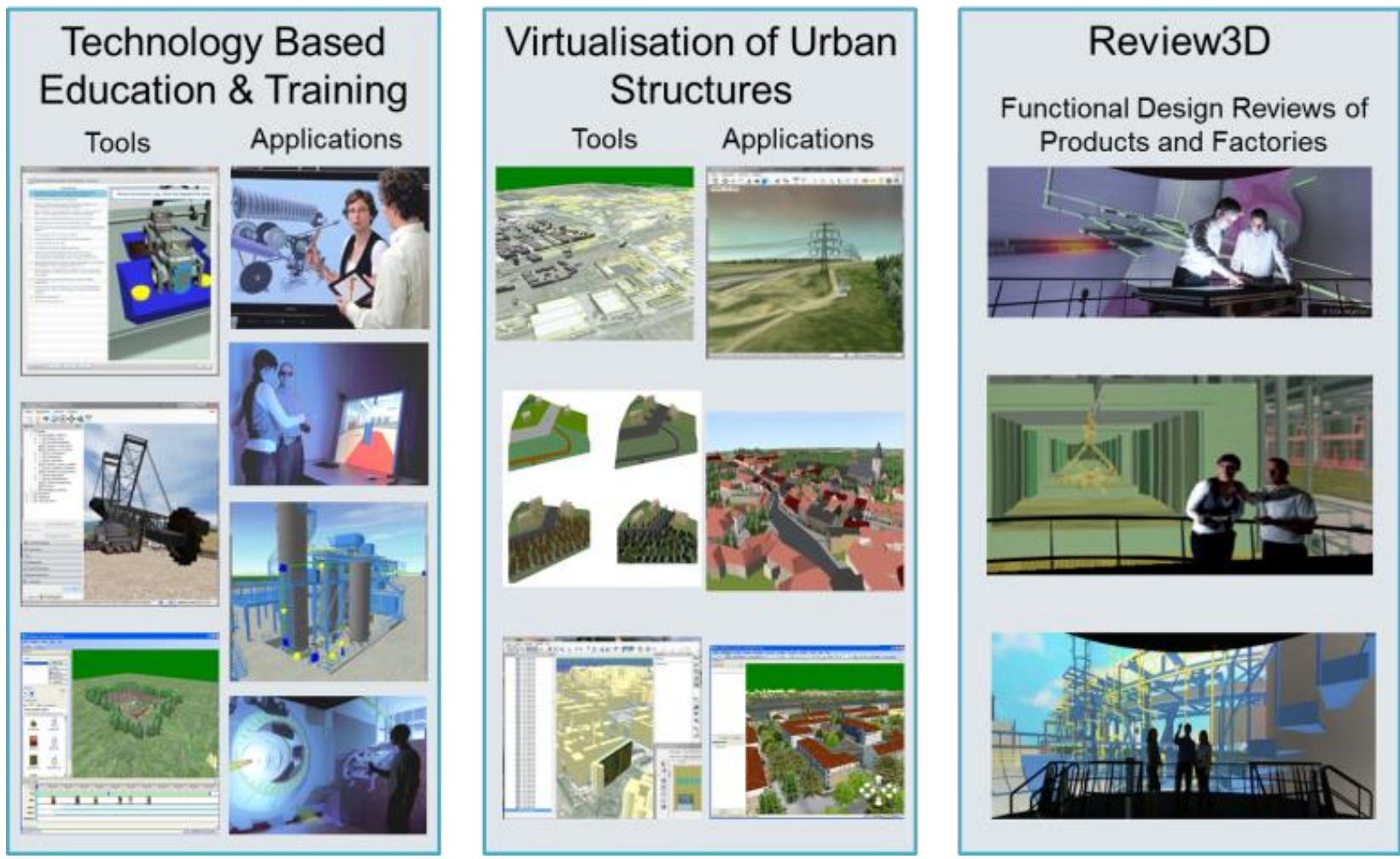

Fig. 2. Fields of application of the VDT Platform, CFraunhofer IFF.

segments as "consumers" of innovative technologies? This paper addresses this issue in the fields of production and logistics and presents examples of applications that highlight prospects for future applications of virtual and augmented reality technologies.

\section{VIRTUAL REALITY TECHNOLOGIES}

Technologies such as 3D visualization, simulation, digitization, virtual reality (VR) and augmented reality (AR) hold a key position in today's information society. These technologies are employed whenever information on, knowledge of and experience with complex systems is transferred.

Virtual reality is a high-end human-computer interface that links real-time simulation and interactions through multisensory channels (sight, hearing, touch, smell and taste) [4]. In its ultimate form, virtual reality enables humans to become part of and to act in a computer generated environment.

In addition to "pure" virtual reality, mixed forms exist, which are subsumed under the term "mixed reality". Mixed reality denotes environments or systems, which combine the real world and virtual reality with each other. In this sense, reality and virtual reality constitute the extremes in a reality-virtuality continuum. The approaches produced are discussed in relation to learning systems in detail in [5]. Bruns speaks of mixed reality when the boundary between the real and virtual parts of a system can be freely drawn user-centrically.

Forms of mixed reality are produced by enriching the real world with virtual components for the purpose of studying real objects (augmented reality) or by enriching the virtual world with real components for purposes of examining the virtual world (augmented virtuality).

One of today's challenges is to apply digital data to create a digital process model of the operating phase. This will enable people to experience digital models of a product or factory environment in a virtual environment, to interactively change it and to immediately receive a feedback of the consequences of these changes. Obviously, such an environment also provides new methods of learning and understanding complex technical systems.

Efficient support of qualification and training in the field requires VR work systems that integrate the aforementioned virtual technologies. It is crucial that the design and simulation tools implemented in the process chain have coordinated interfaces and, thus, data are consistently integrated.

In this form, virtual reality is well on its way becoming an integral part of corporate operations as an information and communication technology. For qualification and training in the field, this means that real work systems for teaching and learning will increasingly be supplemented or replaced by VR work systems. This will necessitate incorporating didactic methods in VR/AR technology development concepts (see [6]).

The Virtual Development and Training (VDT) Platform (Fig. 2) of the Fraunhofer IFF is a software platform that facilitates interaction with realistic virtual products, machinery and plants on the basis of immersive 3D virtual environments. Fields of application for the VDT Platform include product and process development, and education and training.

This virtual interactive system created by the Fraunhofer IFF offers the opportunity to support new ways of communication and collaboration in development and planning processes. In 
the age of digital engineering, the solutions of the Fraunhofer IFF build bridges between established development methods and innovative digital tools.

\section{DigITAL ENGINEERING SOLUTIONS}

Linking together digital tools coming from different domains with the goal to experience a future product and its functionalities in an interactive virtual environment leads to the definition of digital engineering:

Digital engineering refers to continuous application of digital methods and tools throughout the entire product emergence process and the production process. It aims at an improved quality of the planning process and the mastering of all subsequent processes. Digital engineering not only includes the physically correct modeling of all relevant product characteristics, but also the software related functionalities (e.g., functions of embedded systems). The interoperability of all tools used is an essential prerequisite for the early simulation of the production processes within the planning phase as well as for the accompanying simulation of the productions phase. Interoperability can be distinguished into a technical layer (e.g., compatibility of communication protocols), into a semantic layer (compatibility of data exchanged between different tools), and into an organizational layer (integration of digital tools into the work flow). Models and tools need to be designed for an interactive feedback and for the application in a virtual interactive environment. Interoperable digital engineering tools can be combined to form interactive models that approach all human senses necessary to support a specific decision process. These systems are characterized by the following trends:

\section{A. Trend: Realization of Product Functionality by Use of Software Tools}

In addition to control greater product diversity, it is also recommended reducing the time-to-market of products - the time needed from the first idea to a marketable product. This leads to an expansion of the established methods of product and process development about software engineering. Furthermore, a comprehension about how software determines nonfunctional product properties is needed - e.g., product safety and reliability.

\section{B. Trend: The Shortened Product Life Cycle and Increasing Product Variety also Require an Increasing Flexibility of the Means of Production}

This challenges the manufacturer of means of production to consider the final operating modes already in the planning process. It includes remodeling and dismantling variants and making it necessary to consider requirements of future operators and users. Therefore, these variants already have to be simulated during the development process or rather offering this option to the customer.
C. Trend: Beyond Product Manufacturing Today It Is also Essential to Develop and Offer Services

As an implication of this, it is no longer sufficient to respect only functional product properties during the product emergence process. The consideration of logistical aspects or rendered quality-of-service levels and business models for services involving the users is also recommend.

D. Trend: The Conception Design of New Facilities (e.g., in the Field of Process Engineering) Based on Modularized Components to Adapt It on the Fluctuated Market and Making It Energyefficient

The possibility of different systems modes is needed to realize an energy-efficient operation. These operating conditions already had to be considered and if necessary simulated during the planning process. Therefore, explicit data about the operating modes (e.g., startup or shutdown modules) are required. Expert knowledge from control engineers, process engineers and power engineers has to be used at an early stage of the planning process. Thus, the digital tools have to be online capable.

\section{E. Trend: There Is a Registered Increase Complexity of Methods and Tools in Many Operative Ranges. This Requires Collaboration with the Engineering Sciences}

The development and use of new technical devices (e.g., a computer-aided turning center) make it necessary to develop new methods of vocational training and further industrial education for their use. This means for the engineer to transfer classical methods, models, tools and data into other fields of application (e.g., for an operator training in the virtual reality [7], [8]).

In summary of these trends, three main conclusions can be drawn:

- It is necessary to expand virtual engineering to new domains.

- The results of the planning process can be further optimized if data about the subsequent operating modes are early available. This can be achieved using digital models. This requires interoperability to connect the used simulation tools and also real-time ability to support the interactivity and connectivity of real hardware for gradual virtual commissioning.

- The integration of interdisciplinary knowledge is increasing. This requires the inclusion of new user groups (e.g., inclusion of future operators in the planning process). Partly very different digital tools have to be bridged by using a combination of new media for the presentation and interaction in virtual environments. Therefore, compared with previous applications of virtual engineering major expansions are necessary, which are shown in Fig. 3. 


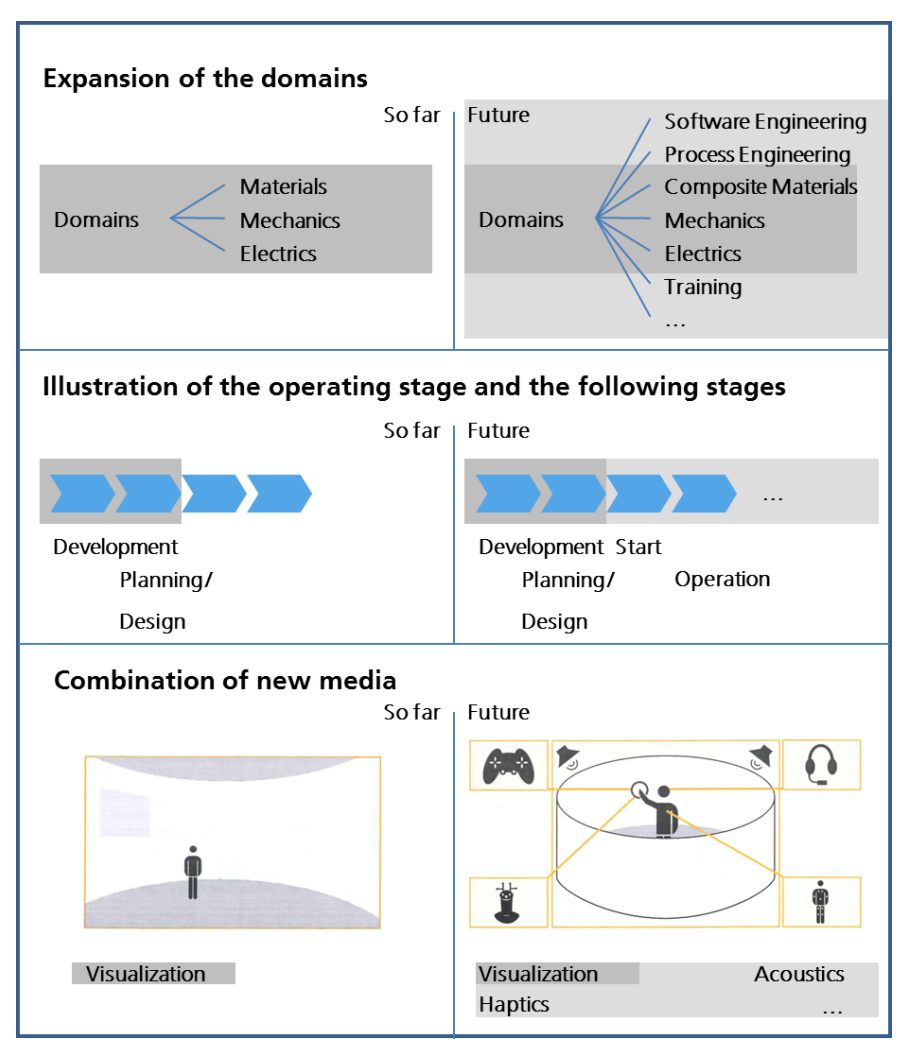

Fig. 3. New approaches of digital engineering, OFraunhofer IFF.

The motivation behind the development of the VDT platform was to create and to establish a platform, which efficiently created and communicated virtual content, which was hitherto only used for presentation purposes. This VR framework provides the ability to perform cross-domain feasibility studies in machinery manufacturing, plant manufacturing, shipbuilding, factory simulation and building design.

\section{EU RELATED RESEARCH PROJECTS}

\section{A. ManuVAR}

The ManuVAR project was launched in May 2009 under the EU 7th Framework Program. This project demonstrates that bundled expert knowledge in business companies can improve the competitiveness of EU industries.

Offshoring is a natural phenomenon. It will exist while there are large differences in labor costs. However, European industries could focus on the business that cannot be offshored - unique or deeply customized products, maintenance of fixed installations, operation and maintenance of machinery. This business relies on the knowledge and skills of people rather than on minimizing labor costs. ManuVAR has contributed to highlighting how manual work can be an opportunity for Europe.

The goals of ManuVAR are to develop an innovative technology platform and a framework to support and improve the whole product lifecycle. ManuVAR focuses on the fields of ergonomics, safety, work assistance and training issues. It includes various people from designers to factory workers, logistics experts, operators, maintenance personnel and endusers.
The key objectives of ManuVAR are the following:

- To improve EU industry competitiveness via knowledgebased business models;

- To increase productivity and quality, and to reduce cost;

- To reduce the need for global outsourcing;

- To support efficient knowledge and skill management through the lifecycle;

- To facilitate adaptation to customized products and customer services.

The ManuVAR consortium has developed the following instruments to improve manual work:

Technological: the platform of reconfigurable and modular virtual and augmented reality tools, system architecture, tested technical prototypes;

Methodological: the ManuVAR PLM model, its application methodology, framework of ergonomics methods to support manual work throughout the system lifecycle;

Policy: recommendations and evidence for policy makers (industry, community, political) that high knowledge and high value manual work can be an opportunity for European industries.

The results made during ManuVAR can be adapted to other industries and service sectors. The need to succeed in global competition is essential for any global acting company, especially in the field of logistics and transportation.

\section{B. ProWis}

Large companies in particular have recognized the importance of the resource knowledge and the necessity of managing it. Since small and medium-sized enterprises (SMEs) have hardly made this issue a priority yet, the Federal Ministry of Economics and Technology of Germany (BMWi) has launched the initiative "Fit for Knowledge Competition", which also supports the project ProWis.

The objective of ProWis was to develop an approach to knowledge management suitable for SMEs and put it to use in SMEs. In the first phase, an approach consisting of five phases was developed. It centered on two proven methods of analysis, which were simplified in the project. The approach was tested by fifteen SMEs and "readied for üproduction". A collection of knowledge management solutions, the ProWis-Shop containing approximately fifty methods and tools, was additionally developed.

In the second phase, the deliverables were transferred to practice to a greater extent. The approach was taught in seminars. Companies exchanged views on potential approaches and formulas for success at networking events, e.g., ProWis Connect. The ProWis Shop has also been developed further and now contains not only descriptions of methods but also concrete instructions on implementing knowledge management and aids to apply the methods of analysis. The ProWis Shop's range of solutions is available to anyone interested free of charge.

The resource knowledge is considered to be the fourth production factor. According to current studies, its optimal use is instrumental to business success. Companies must have fast access to available knowledge and related information, constantly refine it and protect it from loss. Knowledge 
management encompasses all of the systems, methods, instruments and tools, which facilitate systematic treatment of knowledge in all of a company's units. The Fraunhofer IFF's approach is applied and starts with a company's value adding processes to attain improvements.

Process-oriented and integrated knowledge management is implemented in five steps:

Initialization: Since the cornerstone for success is laid at the start, a pilot unit is selected, a team is assembled and a sponsor from management is sought.

Analysis: Employees from the company or a pilot unit are surveyed quantitatively and processes are analyzed qualitatively to identify potentials for improvement and effective approaches.

Goals and solutions: Goals are specified concretely based on the results of the analysis and fields of actions are prioritized before concrete solutions (methods, IT tools) can be selected.

Implementation: Once the individual steps have been planned, the selected knowledge management methods or tools are implemented. The implementation phase includes training sessions or supporting communication actions (internal marketing).

Evaluation: Success is assessed at the end of the project base on the goals set. If necessary, follow-up improvement actions can be devised and initiated. The scope of our support hinges fully on specific needs and prior experience and can be scaled accordingly. A collection of web-based knowledge management solutions has also been developed within the project.

\section{VIDET}

Virtual Reality shortens and simplifies the development of new products. The innovation cluster Virtual Development, Engineering and Training (VIDET) enhances the availability of VR-technologies for mechanical and plant engineering in Saxony-Anhalt, Germany.

Therefore, the innovation cluster VIDET bundles the expert knowledge of the Fraunhofer IFF, the Otto von Guericke University Magdeburg and several regional companies of mechanical and plant engineering.

New products are common developed by using computer technologies. They are digitally designed, constructed, tested and enhanced. By using computers for test purposes, mechanical engineers are able to prove new machines without building expensive prototypes. The interactive 3D model allows running virtual production processes to discover possible failures or potential weak spots and to train the staff. New methods and technologies such as digital engineering and virtual reality make it possible.

The innovation cluster VIDET will enhance the already existing economic potentials of engineering and process industry, will promote innovative product ideas and will support the safeguard employment.

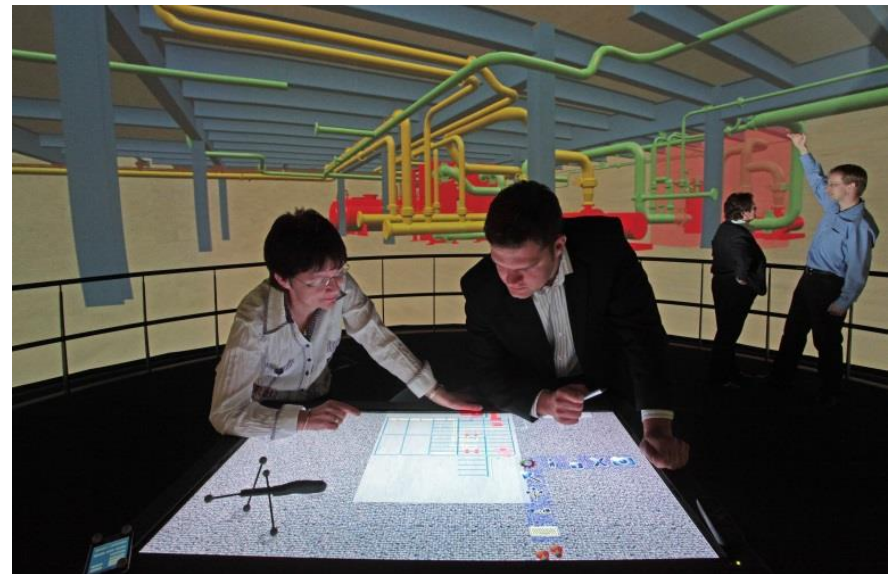

Fig. 4. VR technologies for plant engineering, OFraunhofer IFF, Dirk Mahler.

Therefore, VIDET provides a regional Virtual Reality-based platform for research and business to collaborate on the development of new products, their manufacturing and transportation for the regional industry. Apart from the support of efficient industrial production via virtual process design, the cluster partners of VIDET help to establish innovative technologies for logistics, maintenance and qualification especially in SME.

For instance, the researchers of Fraunhofer support the development process of equipment by using virtual models from CAD systems. To reduce the development time of equipment, the commissioning phase can be virtualized in order to perform functional tests early as possible without completing Design Engineering. By using a virtual prototype, several development tasks can proceed simultaneously (Simultaneous Engineering). Digital models provide the opportunity to start functional tests while the machine is still being manufactured.

Beyond the development process, all information about the product model can be used for diverse applications, e.g., operator training or CNC programmer training as well as the shipping process.

Moreover, VIDET considers that interactive visualization and simulation are increasingly growing in importance for marketing and sales. In the planning and design phase, a machinery manufacturer's client can be involved in the broader development and production process so that the product is customized for a client's specific needs. Being adapted for industries in general means an enormous increase of client enthusiasm based on realistic visualization via virtual models.

\section{ViVERA}

Virtual and augmented reality technologies have made enormous advances in recent years: Germany holds a leading position internationally.

The project acronym ViVERA stands for "Virtual Competence Network for Virtual and Augmented Reality". The network combined ten institutes and universities' research resources in the field of virtual and augmented reality nationwide. 
ViVERA sets itself the task of networking developers and users' expertise and experience and sustainably integrating virtual technologies in business and industry. Among other things, demonstrators were prototyped, which demonstrate the potential and diverse potential applications of VR and AR technologies in the widest variety of fields.

Research results are documented in a knowledge base and integrated in the international research scene. They are, thus, made accessible to a wide circle of potential users. The Virtual Development and Training Centre of the Fraunhofer IFF serves as a contact for technology transfer and establishes contact with the cooperating research partners.

The excellence network of ViVERA is equipped with extensive experiences from already completed projects. The associates are developing customized applications together with their clients from the business communities. The researchers provide consulting when virtual technologies are being implemented in companies and develop individual software solutions completely made-to-order [9], [10].

\section{SAMPLE FOR SUCCESSFUL TECHNOLOGY TRANSFER}

As one result of the technology transfer concept developed within the eINTERASIA project, a virtual reality laboratory at JSC “Astana Innovations" has been created in conjunction with the Fraunhofer IFF. The laboratory is equipped with modern systems of virtual reality, e.g., a CAVE, a multi-screen projection system for virtual reality. To develop different scenarios in the laboratory, Astana Innovation uses the VRframework developed by the Fraunhofer IFF - the VDTPlatform.

Meanwhile major projects have been worked on in the field of virtual reality by using the virtual reality laboratory of Astana Innovations.

\section{A. Virtual Model of Astana}

The virtual model of Astana city is part of the project "Smart Astana". The main objective is to map the territory and infrastructure of Astana in three-dimensional form. Platform for the implementation of the project "Virtual Astana" is a software framework that creates a virtual model of the city using a variety of modeling techniques and visualization.

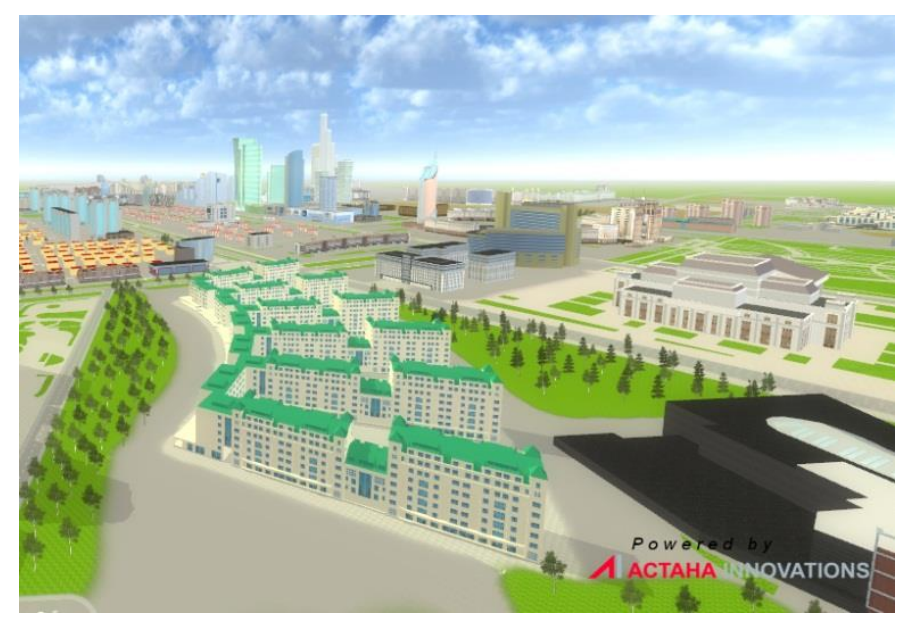

Fig. 5. Virtual model of Astana city, CAstana Innovations.
The development of the virtual model was subdivided into several stages. The first stage was the collection and analysis of baseline information. A wealth of information such as plans of buildings and structures as well as photos were examined and adjusted for their re-use. The linkage of different data types generated a comprehensive analysis of the reconstructed part of the city. Building information like the size, proportions and the ground connection became the basis for the second phase of the virtual model development - creating a library of virtual environment elements.

Processed by computer support, various data have been made compatible with each other, e.g., maps were scanned and digitized. Based on the collected information, typical projects or new buildings were created as elements of the virtual environment of Astana city. Last stage is the coupling of all single library elements into a uniform VR-environment. To place all 3D-objects on the digital map, they were connected with georeferenced data of the city.

Each city has its own unique appearance and, therefore, a set of further realistic 3D-objects, e.g., buildings, roads, parks, squares, bodies of water, fences, flower beds, benches, lights and monuments, was applied.

After modeling the infrastructure of the city, the 3D-models were imported into VDT-Platform. Then the virtual model of the city can be explored in the virtual reality system CAVE.

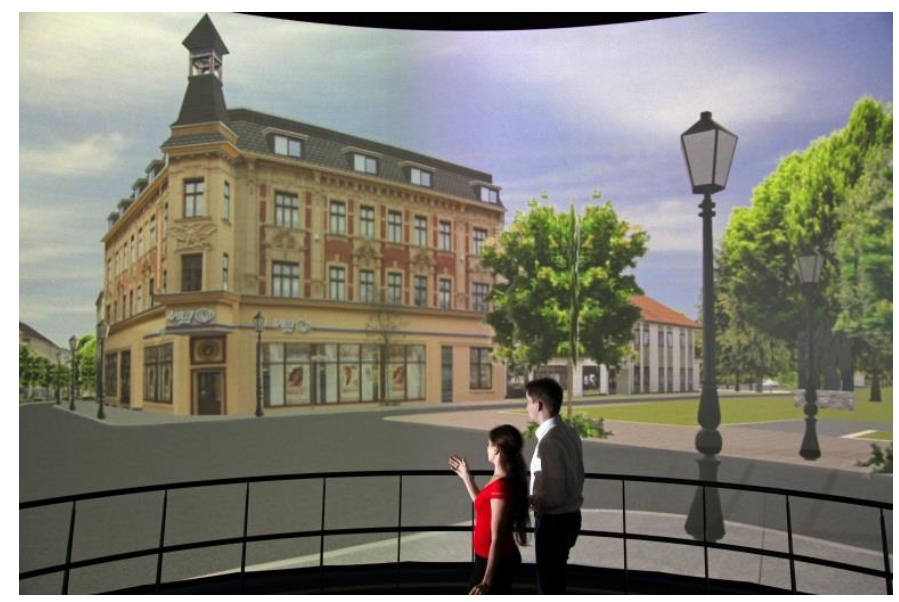

Fig. 6. Virtual city tour in a VR system, CFraunhofer IFF, Dirk Mahler.

\section{CONCLUSION}

The practical approbation of virtual reality models and digital engineering solutions shows the high applicability of virtual reality solutions for a wide range of applications. The increasing availability of 3D models in diverse domains, advanced methods of virtual reality and high performance graphic hardware have established ideal conditions to communicate even very complex knowledge and know-how realistically, rapidly and sustainably. Visualization recognition value, options for realistic interaction with models and the realism of models simulated performance all play a major role. Concepts and tools are essential, which provide users as experts in their fields a user friendly and immersive VR scenario but without deeper knowledge of computer science. 
The use of virtual reality will be fundamental in the age of digital engineering to achieve an effective collaboration between applied research and industry. To achieve a wide and more effective use of VR technologies following the latest trends, it is very important to create appropriate conditions and to promote a cross-sectoral innovation culture in the private and public sector. The development of cross-sectoral turn-keys has to be realized via cooperation through international partner networks of consulting, science \& research and technology. This community of international innovation partners is the basis for the sustainable use of VR technologies to build bridges between established procedures and open innovation.

\section{ACKNOWLEDGEMENT}

The research has been funded by the 7 th Framework Program of the European Commission and is part of the project: reference "600680", title "ICT Transfer Concept for Adaption, Dissemination and Local Exploitation of European Research Results in Central Asian Countries".

\section{REFERENCES}

[1] Belardinelli, C., Blümel, E., Müller, G., Schenk, M., "Making the virtual more real: research at the Fraunhofer IFF Virtual Development and Training Centre," J. Cognitive Processing, vol. 9, pp. 217-224, 2008. http://dx.doi.org/10.1007/s10339-008-0216-0

[2] Blümel, E., "Virtual Reality Based Technology Platforms for Development, Testing and Training," Ginters, E, Bluemel, E., (eds.), in Proceedings of Annual International Conference Virtual and Augmented Reality in Education, VARE 2011, Riga, Latvia. Vidzeme University of Applied Sciences, pp. 1-16, March 18, 2011.

[3] Blümel, E., Fredrich, H., Winge, A., "Applied knowledge transfer to European SMEs by expertise networks using mixed reality," Niedrite, L, Strazdina, R., Wangler, B., (eds.), BIR 2011 International Workshops and Doctoral Consortium, Oct. 6, 2011, Riga, Latvia. Revised Selected Papers. Lecture Notes in Business Information Processing. Berlin Springer, vol. 106, pp. 90-101, 2012. http://dx.doi.org/10.1007/978-3 642-29231-6_8

[4] Burdea, G., Coiffet, P., Virtual Reality Technology, 2nd ed., John Wiley \& Sons Inc, 2003.

[5] Bruns, F.W., "Lernen in Mixed Reality," ABWF (ed.) Kompetenzentwicklung, 2003, pp. 71-112, Waxmann, Berlin, 2003.

[6] Blümel, E., "Stand und Entwicklungstrends des Einsatzes von VR/ARTechniken für Qualifizierung und Training im Arbeitsprozess“". In: GfA e.V. (Hrsg.), Kompetenzentwicklung in realen und virtuellen Arbeitssystemen, pp. 241-244, GfA-Press, Dortmund, 2007.
[7] T. Reek, A. Winge "Realistic virtual systems to improve vocational training". in Achievements and Results 2009 Annual Report of the Fraunhofer Institute for Factory Operation and Automation IFF Magdeburg, pp. 96-97, Magdeburg, 2009.

[8] Bluemel, E., Fredrich, H., Winge, A., "Using Mixed reality for expertise networks to transfer knowledge to industry," in Perspectives in Business Informatics Research. Local Proceedings of the 10th Int. Conf., BIR 2011, Associated Workshops and Doctoral Consortium, Riga, Latvia, pp. 181188, Oct. 6-8, 2011

[9] Fraunhofer IFF, "Virtual Development and Training Centre VDTC Technology and Research" May 2015. [Online]. Available: http://www.vdtc.de/en/technology-vdtc/projects-vdtc/vivera-virtualreality-augmented-reality.html. [Accessed: May 19, 2015].

[10] ViVERA, "Home page - ViVERA", May 2015. [Online]. Available: http://www.vivera.org. [Accessed: May 19, 2015].

Marco Schumann is the Head of the Business Unit of Virtual Interactive Training (VIT) from the Fraunhofer IFF. He graduated from the Otto-vonGuericke University Magdeburg in 1998 with a Master Degree in Computer Science and Specialization in Computer Graphics and Simulation. He started his career as a scientific employee at the Fraunhofer Institute for Factory Operation and Automation IFF in Magdeburg, Germany. Becoming a Project Manager in 2004, he also contributed to the establishment of the Virtual Development and Training Centre (VDTC) of the Fraunhofer IFF in 2006. At the VDTC he led several national research projects funded by the German Ministry for Research and Education and numerous applied research projects with industrial clients. He received his Doctoral Degree from the Otto-vonGuericke University in Magdeburg, the Department of Mechanical Engineering in 2010. His primary research interest is the application of simulation methods in interactive virtual environments. Address: Sandtorstrasse 22, 39106 Magdeburg, Germany, Phone: +49 3914090 158, Fax: +49 3914090115.

E-mail: marco.schumann@iff.fraunhofer.de

Stefan Leye holds a Diploma in Mechanical Engineering (2011). Primary field of study - integrated product development. Currently he works at the Fraunhofer Institute for Factory Operation and Automation IFF, Magdeburg Germany, as a Project Manager at the business unit Virtual Interactive Training. Main field of research: Virtual Reality and Digital Engineering solutions for product development and training. Address: Sandtorstrasse 22, 39106 Magdeburg, Germany, Phone: +49 3914090 114, Fax: +49 3914090115. E-mail: stefan.leye@iff.fraunhofer.de

Artyom Popov holds a Diploma in Electrical Engineering (2008). Primary field of study - electrical systems and grids. Currently he works at JSCAstana Innovations, Astana - Kazakhstan, as a Project Manager at the Laboratory of Virtual Reality. Main field of research: Virtual Reality and Digital Engineering solutions for product development and training. Address: 010013, RK, Astana, Esil area, Dostyk Street 13, Nursaya 2 residential complex. Phone: +7717257 8994.

E-mail: a.popovqmail.ain.kz 